\title{
Electricity Market Price Assesment Utilizing Hybrid PSO - ANN Algorithm
}

\author{
Anamika, Kiran Srivastava
}

\begin{abstract}
Prediction of cost is the most imperative task and the reason for settling on choices in competitive bidding strategies. Reliability, Robustness and optimal benefits for the market players are the fundamental concerns which can be accomplished by a point value anticipating module constitute of diminutive prediction errors, reduced complexity and lesser computational time. Thus in this work, a coordinated methodology dependent on Artificial Neural Networks (ANN) prepared with Particle Swarm Optimization (PSO) is proposed for momentary market clearing costs anticipating in pool based electricity markets. The proposed methodology overcomes the difficulties like trapping towards local minima and moderate convergence as in existing techniques. The work was speculated on territory Spain electricity markets and the outcomes obtained are compared with hybrid models presented in the previous literature. The response shows decline in forecasting errors that are recognized in price forecasting. The total research may help the ISO in finding the key factors that are fit for expectation with low errors.
\end{abstract}

Index terms: Artificial Neural Networks; Particle Swarm Optimization; Market clearing price

\section{I.INTRODUCTION}

Introduction of deregulation leads to a customer driven, organized electricity market, which offers opportunities for optimal resource utilization and efficient electricity procurement strategy. The main aim of organized electricity market is to shrink the cost of electricity through competition and maximize the efficient generation and consumption of electricity $[1-3,28]$. The accurate price prediction has become important in the new restructured electricity market [4], as it plays a key role in power system plans, risk assessment and other related decisions [5-6]. Because of the dissipate nature of electricity, all the generated electricity must be expended. This initiates the need of accurate price forecasting methodologies for both producers and consumers to establish their own strategies for the benefit or utilities maximization [7]. Popularity of forecasting approaches is based on their accuracy, flexibility, reliability and robustness. Choice of most instructive value credits to address market price conduct and determination of fitting estimating model fit for foreseeing the value utilizing gave information are two main decision which ought to be made viably in determining market costs.

The prediction of future price is based on various parameters which include forecasted electricity demand, temperature, sunshine, fuel cost, and precipitation. Presently,

Revised Manuscript Received on November 25, 2019.

Anamika, Electrical \& Electronics Engineering Department, Galgotias College of Engineering \& Technology, Greater Noida, India E-mail; anamika17@galgotiacollege.edu

Kiran Srivastava, Electrical \& Electronics Engineering Department, Galgotias College of Engineering \& Technology, Greater Noida, India many attributes are available for a day-ahead electricity price prediction. Many factors influence the spot prices and can be considered as input features for forecasting the prices. Some of the input features may be historical load, system load rate, historical reserves, forecasted load, forecasted reserves and historical prices [11]. Day, time slot, estimated demand, load change and prices of a day before one week before, two weeks before, three weeks before and four weeks before are considered as inputs for price forecasting [12]. The aggregation of load, surplus, historical market clearing prices and oil prices are used as input data set for the price forecasting [13]. The results show that average and maximum day-ahead load and the day-ahead surplus are the most important inputs for forecasting prices.

There are many approaches that can be observed in the electricity price forecasting. The next day electricity prices are evaluated in view of time arrangement models like transfer function and dynamic regression models [9]. Non parametric functional methods are proposed to forecast the electricity price and demand series in the market of mainland Spain [19]. In [20], a mid-term electricity price forecasting model using multiple support vector machines (SVMs) is proposed to forecast the hourly electricity price for entire 6 months ahead. In this two SVM modules are used separately for both classifying and forecasting the prices. The work is improved and the SVM module is adjoined with auto regressive moving average with external input (ARMAX), to re-define the error values that obtained from the Least Squares SVM (LSSVM) [14, 15]. The contribution of wavelet transform is great in hybrid models used for forecasting the electricity prices. The wavelet transforms decomposes and reconstruct the ill-behaved prices into a set of better behaved price series. Hence, these are combined with auto regressive integrated moving average (ARIMA) and generalized autoregressive conditional heteroskedasticity $(\mathrm{GARCH})$ to forecast the highly volatile and highly nonlinear electricity prices. [7]. The GARCH models are replaced by particle swarm optimized (PSO)-LSSVM forming a new method which is hybrid and based on wavelet-ARIMA-LSSVM to enhance the prediction rightness for the price forecasting [8] The wavelet transform with ARIMA model is combined with Radial Basis Function Neural Network (RBFN) to enhance and overcome the disadvantages in the previous combinations [23].

In [16], a hybrid evolutionary adaptive (HEA) model is developed to forecast the electricity prices in the short term pool based electricity markets. Different methodologies like Wavelet Transform (WT), Evolutionary PSO (EPSO) and Adaptive Neuro Fuzzy Inference Systems (ANFIS) are combined to form HEA. This hybrid model had shown 


\section{Electricity Market Price Assesment Utilizing Hybrid PSO - ANN Algorithm}

an average of $4.18 \%$. An integrated model based on WT, LSSVM and chaotic gravitational search algorithm (CGSA) is used for prediction of the electricity prices in Spanish, Iran's and Ontario's electricity markets. The computational time required for this method to produce the results is 27 minutes which is the major disadvantage of the integrated model. Similarly, a hybrid approach constituting of PSO ANFIS is employed to predict the prices [10]. The hybrid and integrated methodologies have certain advantages and disadvantages. The major challenges faced by these methodologies are consumption of time and complexity in the application.

The shortcomings faced by hybrid and integrated models in the electricity price forecasting can be overcome by a new branch of simplified techniques. ANNs got weighted attention due to its simplicity, accuracy along with a high degree of learning capability in a very short duration of time. Especially, ANNs are getting prominent roles in predicting the electricity prices and demand series. These are successfully applied to forecast the electricity prices by using 13 parameters as inputs [12]. Simple ANNs are applied to forecast the electricity prices of cold and warm days with low, normal and peak loads [21]. A review on electricity price forecasting is presented based on various methodologies used and factors influencing the electricity prices. The various methodologies include game theory models, simulation models and time series models [22]. Neural networks are combined with wavelet transforms and fuzzy logic to predict the signals with hard non linear behavior and time variant functional relationships [24]. Enormous quantities of this present reality input information samples to train artificial neural networks, typically makes confusion over artificial neural networks, during the learning procedure and thus degrades their predictive capacity.. Hence, discrete cosine transform (DCT) is applied as a preprocessing tool for neural networks (NNs) in the estimation of power cost. The time series of costs are changed into frequency domain using DCT and these are then passed through NN for the prediction [18].

The literature survey on various forecasting models reveals that the forecasting methods follow either time series models or hybrid models or neural networks. The time series models are linear and are hard to predict the non-linear price series. The hybrid models are time consuming and complex in usage. The neural networks combined with DCT and modules of wavelet uses repeated normalization of the historical times series which are used as training data set for the ANNs. Slow convergence rate and tendency to become trapped in local minima are the drawbacks of back propagation algorithm (BPA) used in training the NNs.

Hence, to overcome the complex linear models, slow and time taking model with repeated normalization approach , trapping and slow convergences ANN training with PSO is in this research work to forecast the next 168 hours power in test markets for the weeks in year 2002. Further, the structure of the paper is as follows: Section 2 provides research methodology and data required for electricity price forecasting. The proposed research algorithm is represented in section 3. Numerical results and comparative analysis are explored in section 4 and concluded milestones of the research work are presented in the section 5 .

\section{II.METHODOLOGIES}

\section{A. Collection of Data}

The historical prices from Spanish Electricity market are considered to perform the proposed work. Huge number of factors may influence the electricity price such as, system load of the full covered area, power import and export, assessable hydro energy and fuel prices [12]. Out of several parameters proposed by many researches, the historical prices are considered as the most suitable input parameters for the analysis.

\section{Historical Prices}

The different price lags are considered as input parameters for the proposed research work. The different price data are taken from daily trading reports of mainland Spain [22]. The reports constitute of hourly market clearing prices for the Spanish electricity market.

The various inputs considered for this work consist of one week historical electricity price data of Spanish electricity market, i.e. three consecutive previous hour prices, two hourly consecutive prices of previous day, two consecutive prices of two days before, similar hour prices of three days before, same point prices of fourth, fifth and sixth days before and the same hour price of one week before.

These data are taken as information highlights for every hour of the day for both testing and training data sets $[11,12$, 16, 17, and 18].

\section{B.Forecasting electricity prices using integrated approach}

Artificial Neural Networks have been extraordinarily used in wide areas of research like classification, function approximation, pattern recognition, prediction and optimization. In general a Multi Layered Perceptron (MLP) network consists of 3 layers; namely are input, hidden and output layers [12 and 28]. The fundamental step in accurate estimate of any nonlinear mapping can be accomplished by sound training of the neural system. Generally the ANN is trained based on the statistical parameters, which are preprocessed. The output of the neurons can be determined as shown below.

In general, the ANNs learn through updating weights and biases based on BPA by minimizing mean squared error between actual and targeted outputs. In this study, a three layered model of ANN is utilized to get good accuracy of the proposed scheme by training with PSO algorithm. A large testing data set is presented to check the solution. The set of

completely unknown test data was applied for validation and testing.

\section{C.PSO Algorithm}

PSO is an evolutionary approach proposed by Kennedy and Eberhart in the year 1995 [29]. It is inspired based on the biological and sociological behavior of birds searching for their food. In searching the path for the food, best solution is also captured. It initiates by placing the particles randomly in problem hyperspace. Consider a variable problem with particle in a swarm. The 
vectors and of a particle denotes the position and velocity respectively. The position of particles were updated based on current position, current velocity, distance from and . The updations of velocity and position vector are done based on the following rules:

$$
v_{i}^{t+1}=w v_{i}^{t}+c_{1} \times \operatorname{rand} \times\left(p_{\text {best }, i}-x_{i}^{t}\right)+c_{2} \times \operatorname{rand} \times\left(g_{\text {best }}-x_{i}^{t}\right)
$$

$$
x_{i}^{t+1}=x_{i}^{t}+v_{i}^{t+1}
$$

Here,

$$
\begin{array}{ll}
v_{i}^{t} & \text { Velocity of particle } i \text { at iteration } t \\
w & \text { Weighting factor } \\
c_{j} & \text { Acceleration coefficient } \\
\text { rand } & \text { Random number between } 0 \text { and } 1 \\
x_{i}^{t} & \text { Position of particle } i \text { at iteration } t \\
p_{\text {best, } i} & \text { Local best of swarm } i \text { at iteration } t \\
g_{\text {best }} & \text { Best solution so far }
\end{array}
$$

In the equation (4) $w v_{i}^{t}, c_{1} \times \operatorname{rand} \times\left(p_{\text {best }, i}-x_{i}^{t}\right)$ and $c_{2} \times$ rand $\times\left(g_{\text {best }}-x_{i}^{t}\right) \quad$ provides exploration ability, individual effort and group effort of particles respectively. In each of the iteration, calculated velocities are employed to find the next position of the particles. This will proceed until the stop basis is met [30]. In the present work maximum number of iterations and least or whichever is the first is used as stopping criteria for the PSO algorithm.

\section{RESULTS}

The proposed PSO trained ANN approach is validated on Spanish Electricity Markets for the year 2002. It is utilized by many of the researchers as a standard point of references. Hence, prices in the year 2002 are used as test case in the present work. A case study for the month of February resembling winter season is considered for the forecasting $[16,17,18,23$ and 24]. The training data set constitutes of hourly prices of 6 weeks past to the day, whose prices are to be estimated. Table 1 shows present scenario, the testing and training data sets for Spanish electricity markets.

Table I

The Various Data Set Used For Training And Testing The Ann For Spanish Electricity Markets

\begin{tabular}{|c|c|c|}
\hline & Training Data & Testing Data \\
\hline Winter & January 7 to February 17, & February 18 to 24, \\
week & 2002 & 2002 \\
\hline
\end{tabular}

The estimated and the actual price values in the Spanish electricity market for the winter week is shown in fig. 1 . The figure reveals the closeness between the actual and predicted values. The figure also reveals that the estimated value follows the actual even at the peaks and off peaks.

The empirical results obtained for the Spanish electricity markets are in comparison with the previous researches employing the statistical indicators. The Mean Absolute Percentage Error ( ) is taken as a statistical indicator to determine the forecast capacity of the proposed approach.

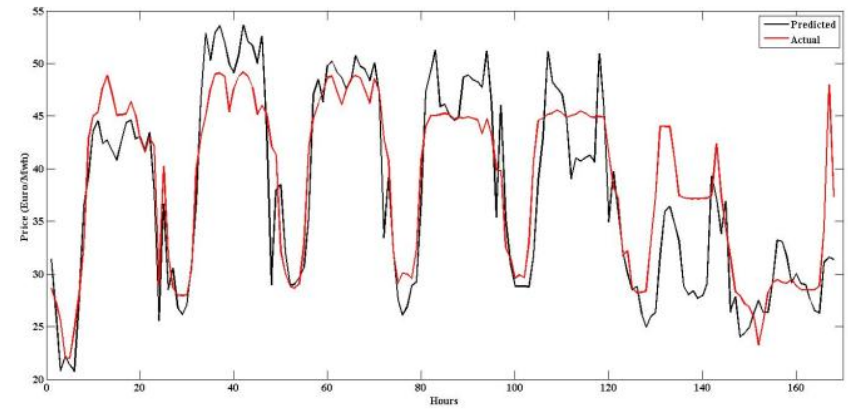

Fig. 2 Graph between the actual and predicted value for the winter months

The MAPE $(\%)$ is expressed as:

$$
\operatorname{MAPE}(\%)=\frac{100}{N} \sum_{i=1}^{N} \frac{\left|y_{i f}-y_{i a}\right|}{y_{i a}}
$$

Table 2 shows results of achieved throughsuggested approach for the Spanish electricity markets. It is examined that the iteration count in PSO makes a huge importance in training the neural networks. It is also noticed that the suggested way to forecast the prices with an average of $2.7641 \%$ at an iteration count of 500. It could be further reduced by increasing the iterations in the PSO algorithm but it is observed that the PSO will take much time in evaluation of prices. Table 3 shows the results of the test week is obtained through other approaches like wavelet transform neural networks fuzzy logic (WNF) [24], wavelet-ARIMA-RBFN [23], wavelet-EPSO-ANFIS [16], CGSA-LSSVM [17] and the suggested technique. The proposed approach produces the results with better forecasting accuracy over the other approaches. An average value of $6.32 \%$ of has been recently reported DCT-ANN [18]. It can be seen, the performance of the suggested method is best when compared to recently proposed techniques. Although, the model is not made for forecasting the price spikes specifically, it performs satisfactorily in their presence with finer overall results.

It is clear from numerical results that the proposed approach can present a more effective and accurate forecasting which are nearer to real Spanish's electricity market prices. The results through the proposed approach are obtained in fraction of seconds using MATLAB on a personal computer with $2 \mathrm{~GB}$ of RAM and $2.0 \mathrm{GHz}$ based processor. The reduced complexity and lesser computational times are the novelties of the proposed PSO pre-processed ANN

approach. The exclusiveness of the suggested approach lies in lesser calculation time in evaluating the prices as compared with many other recent techniques. The necessary calculation timefor the module recently expressed usingLSSVM, wavelet and CGSA [17] is about 27 minutes. The approach detailed based on wavelet-EPSO-ANFIS in [16] needs a minimum 40 secondstime to produce the result. The necessary computational time required by the recently suggested approaches like wavelet- MI+CNEA [23],MI+CNN [25] and ARIMA-RBFN [26], were $40 \mathrm{~min}, 35 \mathrm{~min}$ and $5 \mathrm{~min}$ respectively. But, the proposed approach evaluates the price series with a reduced computational time. Hence

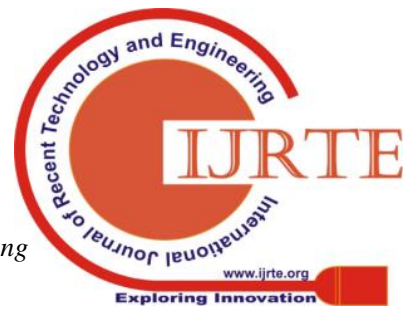




\section{Electricity Market Price Assesment Utilizing Hybrid PSO - ANN Algorithm}

this causes the novelty of the proposed approach and could be used for real life applications to present the best tradeoff between prediction accuracy and computation times.

\section{Table II}

Results Of Through Proposed Approach

\begin{tabular}{|c|c|c|c|}
\hline S. No. & Model & MAPE & Iteration count \\
\hline 1 & ANN with PSO training & $\mathbf{2 . 7 6 4 1}$ & $\mathbf{5 0 0}$ \\
\hline 2 & ANN with PSO training & $\mathbf{2 . 8 3 8 3}$ & $\mathbf{3 0 0}$ \\
\hline 3 & ANN with PSO training & $\mathbf{3 . 0 5 4 7}$ & $\mathbf{2 0 0}$ \\
\hline 4 & ANN with PSO training & $\mathbf{3 . 3 0 4 9}$ & $\mathbf{1 0 0}$ \\
\hline 5 & ANN with LM training & $\mathbf{4 . 2 5 1 6}$ & $\mathbf{2 0 0}$ \\
\hline
\end{tabular}

\section{Table III}

Comparative Results For Spanish Electricity Market Winter Week

\begin{tabular}{|c|c|}
\hline Reference & Winter week \\
\hline Reference [24] WNF & 3.3800 \\
\hline Reference [23] Wavelet +ARIMA+RBFN & 4.2700 \\
\hline Reference [16] HEA & 3.0400 \\
\hline Reference [17] CGSA+LSSVM & 4.4170 \\
\hline Reference [18] DCT-ANN & 4.0300 \\
\hline PSO trained ANN & 2.7641 \\
\hline
\end{tabular}

\section{IV.CONCLUSION}

This paper presents a novel model for day-ahead electricity price forecasting by using the artificial neural networks for Spanish electricity markets. The historical prices for the previous 42 days are considered as the input factors for forecasting the next 7 days. The present work is compared with advanced, hybrid and complex models which are used in the recent researches. The numerical results suggest that the accuracy is well-heeled and most appropriate for forecasting the electricity prices for the deregulated environments in comparison with previously developed models.

\section{REFERENCES}

1. M. Prabavathi, R. Gnanadass, "Energy bidding strategies for restructured electricity market," International Journal of Electrical power and energy systems, vol. 64, pp. 956-966, 2015.

2. Matthew Dornan, "Reform despite politics? The political economy of power sector reform in Fiji," 1996-2013, Energy Policy, vol. 67, pp. 703-712, 2014.

3. SławomirŚmiechn, Monika Papież, "Energy consumption and economic growth in the light of meeting the targets of energy policy in the EU: The bootstrap panel Granger causality approach,” Energy Policy, vol. 71, pp. 118-129, 2014.

4. C. P. Rodriguez and G. J. Anders, "Bidding strategy design for different types of electric power market participants," IEEE Trans. Power Systems, vol. 19, pp. 964-971, 2004.

5. Tebello Mathabaa, Xiaohua Xia, Jiangfeng Zhang, "Analyzing the economic benefit of electricity price forecast in industrial load scheduling," Electric power systems research, vol. 116, pp. $158-165,2014$

6. J. P. S. Catalao, S. J. P. S. Mariano, V. M. F. Mendes, and L. A. F.M Ferreira, "Short-term electricity prices forecasting in a competitive market: a neural network approach," Electric power systems research, vol. 77, pp. 1297-1304, 2007

7. H. Zareipour, C. A. Canizares, K. Bhattachara, and J. Thomson, "Application of public-domain market information to forecast Ontario's wholesale electricity prices,” IEEE Trans. Power Systems, vol. 21, pp. 1707-1717, 2006

8. Jinliang Zhang, Zhongfu Tan, Shuxia Yang, "Day-ahead electricity price forecasting by a new hybrid method," Computers \& Industrial Engineering, vol. 63, pp. 695-701, 2012.

9. Francisco J. Nogales, Javier Contreras, Antonio J. Conejo, Rosario Espínola, "Forecasting Next-Day Electricity Prices by Time Series Models," IEEE Transactions on power systems, vol.17, pp. 342-348, 2002.

10. Pousinho H M I, Mendes V M F, Catalao J P S. "Short-term electricity prices forecasting in a competitive market by a hybrid

PSO-ANFIS approach,” International Journal of Electrical Power \& Energy Systems, vol. 39, pp. 29-35, 2012.

11. S. K. Aggrawal, L. M. Saini, A. Kumar, "Electricity Price Forecasting in Deregulated Markets: A Review and Evaluation," International Journal of Electrical power and energy Systems, vol.31, pp. 13-22, 2009.

12. Deepak Singhal, K. S. Swaroop, "Electricity price forecasting using Artificial Neural Networks," International Journal of Electrical power and energy Systems, vol. 33, pp. 550-555, 2011.

13. Li Zhang, Peter B. Luh, "Neural Network based Market clearing price prediction and confidence interval estimation with an improved extended kalman filter," IEEE Trans. Power Systems, vol. 20, pp. 59-66, 2005.

14. Xing Yan, Nurul A. Chowdhury, "Mid-term electricity market clearing price forecasting utilizing hybrid support vector machine and auto regressive moving average with external input," International Journal of Electrical power and energy systems, vol. 60, pp. 64-70, 2014

15. Xing Yan, Nurul A. Chowdhury, "Mid-term electricity market clearing price forecasting: A hybrid LSSVM and ARMAX approach," International Journal of Electrical power and energy systems, vol. 53, pp. 20-26, 2013.

16. G. J. Osorio, J. C. O. Matis, J. P. S. Catalao, "Electricity prices forecasting by a hybrid evolutionary adaptive methodology," Energy Conversion and Management, vol. 63, pp. 363-373, 2014.

17. H. Shayeghi, A. Ghasemi, "Day-ahead electricity prices forecasting by a modified CGSA technique and hybrid WT in LSSVM based scheme," Energy Conversion and Management, vol. 74, pp. 482-491, 2013

18. S. Anbazhagan, N. Kumarappan, "Day- ahead deregulated electricity market price forecasting using neural network input featured by DCT," Energy conversion and management, vol. 78, pp. 711-719, 2014.

19. Juan M Vilar, Ricardo Cao and German Aneiros. "Forecasting next day electricity demand and price using non parametric functional methods," International Journal of Electrical power and energy systems, vol. 39, pp. 48-55, 2012.

20. Xing Yan, Nurul A. Chowdhury, "Mid-term electricity market clearing price forecasting: A multiple SVM approach," International Journal of Electrical power and energy systems, vol. 58, pp. 206-214, 2014.

21. M. Zarezadeh, A. Naghavi and S. F. Ghaderi, "Electricity price forecasting in Iranian electricity market applying artificial neural networks," IEEE Electrical power and energy conference, 2008.

22. Spanish electricity market web site http://www. omel .com [active January 2016].

23. Amjady N, Keynia F. "Day-ahead price forecasting of electricity markets by mutual information technique and cascaded neuro-evolutionary algorithm," IEEE Trans. Power Systems, vol. 24, pp. 306-318, 2009

24. J. P. S. Catalao, H. M. I. Pousinho, V. M. F. Mendes, "Short term electricity prices forecasting in a competitive market by a hybrid intelligent approach," Energy conversion and management, vol. 52, pp. 1061-1065, 2011

25. Keynia F. "A new feature selection algorithm and composite neural network for electricity price forecasting," Engineering Applications of Artificial Intelligence, vol. 25, pp. 1687-1697, 2012.

26. M. Shafie Khan, M. Paras Moghaddam, M. K. Sheikh-El-Eslami, "Price forecasting of day-ahead electricity markets using a hybrid forecast method," Energy conversion and management, vol. 52, pp. 2165-2169, 2011

27. M. Gori, A. Tesi, "On the problem of local minima in back-propagation,” IEEE Trans. Pattern Anal. Mach. Intell., vol. 14, pp. 76-86, 1992.

28. Anamika, Rajagopal Peesapati, Niranjan Kumar, "Estimation of GSR to ascertain solar electricity cost in deregulated electricity markets,' Renewable Energy, vol. 87, pp. 353-363, 2016.

29. J. Kennedy, R.C. Eberhart, "Particle swarm optimization," In: Proc. IEEE International Conference on Neural Networks, vol. 4, pp. 1942 1948, 1995.

30. Seyed Ali Mirjalili, Siti Zation Mohd Hasim and Hossein Moradian Sardroudi, "Training feed forward neural networks using hybrid particle swarm optimization and gravitational search algorithm," Applied Mathematics and Computation, vol. 218, pp. 11125 - 11137, 2012. 